\title{
Adaptive hybrid impedance control of robot manipulators: A comparative study
}

\author{
Luís F. Baptista, José M. G. Sá da Costa \\ Technical University of Lisbon, Instituto Superior Técnico \\ Department of Mechanical Engineering, GCAR/IDMEC, \\ Avenida Rovisco Pais, 1096 Lisboa Codex, Portugal. \\ Telephone: 351-1-8418190. Fax: 351-1-8498097. \\ email: baptista@gcar.ist.utl.pt / msc@gcar.ist.utl.pt
}

\begin{abstract}
This article presents an adaptive hybrid impedance control approach for robot manipulators. The proposed scheme, consists of an outer hybrid impedance control loop that generates a target acceleration to an inner adaptive controller in order to linearize the robot dynamics and track the desired force/position. In order to analyse the performance of the proposed control scheme, two distinct adaptive control algorithms in the inner control loop are tested. The performance of the hybrid impedance strategy is illustrated by computer simulations with a 2-DOF PUMA 560 robot, in which the end-effector is forced to move along a frictionless surface in the vertical plane. The results obtained for both adaptive algorithms, reveal an accurate force tracking and impedance control in robotic tasks that require force tracking capability in an rigid environment with a time varying stiffness profile.
\end{abstract}

\section{Keywords}

Robotics, hybrid impedance control, direct adaptive control, passivity-based adaptive control

\section{INTRODUCTION}

Control schemes for a robot manipulator in contact with an environment can be classified as open-loop force control or direct closed-loop force control. The first approach classified as impedance control (Hogan, 1985), do not attempt to control force explicitly but rather to control the relationship between force and position of the end-effector in contact with the environment. Controlling the position, therefore leads to an implicit control of force. The second approach, hybrid control (Raibert, 1982), separates a robotic force task into two subspaces, a force controlled subspace and a position controlled subspace. Two inde- 
pendent controllers are then designed for each subspace. In 1988, Anderson and Spong (Anderson, 1988), proposed a new method termed Hybrid Impedance Controller which combines the hybrid control and impedance control strategies, into a unified approach. It separates the task space into two subspaces, an explicit force controlled subspace and an impedance controlled position subspace.

Many researchers have studied the application of adaptive control in robotics, as in the paper by Craig and Sastry (Craig, 1986), where adaptive control is applied to control a robot in free motion, while Lu and Meng (Lu, 1991) and Colbaugh et al. (Colbaugh, 1995) applied adaptive impedance control to a robot in constrained motion.

In this article, the performance of the proposed adaptive hybrid impedance strategy with the algorithms proposed by Colbaugh et al. (Colbaugh, 1995) and by Siciliano and Villani (Siciliano, 1996) respectively, is analysed in order to show the closed-loop capability of the scheme in constrained motion.

This paper is organized into 6 sections. Section 2 summarises the modelling of the manipulator and the environment. Section 3 presents the hybrid impedance approach. Section 4 discusses the adaptive algorithms and the overall control schemes. Simulation results are presented and analyzed in Section 5. Finally, conclusions are given in Section 6.

\section{MANIPULATOR AND ENVIRONMENT DYNAMICS}

A rigid robot in constrained motion is governed by a set of nonlinear dynamic equations, given by

$$
\boldsymbol{M}(q) \ddot{q}+\boldsymbol{c}(q, \dot{q}) \dot{q}+\boldsymbol{g}(q)+\boldsymbol{d}(\dot{q})=\tau-\boldsymbol{J}^{T}(q) \boldsymbol{f}_{e}
$$

where $q, \dot{q}$ and $\ddot{q}$ correspond to the $(n \times 1)$ vectors of joint angular positions, velocities and accelerations, respectively. $\boldsymbol{M}(q)$ represents the $(n \times n)$ symmetric positive definite inertia matrix, $\boldsymbol{c}(q, \dot{q}) \dot{q}$, describes the $(n \times 1)$ vector of Coriolis and centrifugal effects, $\boldsymbol{g}(q)$ accounts for gravitational terms and $\boldsymbol{d}(\dot{q})$ for the frictional terms in joint coordinates, respectively. The term $\boldsymbol{J}$ represent the Jacobian matrix that relates the joint velocity to the linear and angular velocities of the end-effector, the term $\tau$ represents the $(n \times 1)$ vector of applied joint torques and $\boldsymbol{f}_{e}$ denotes the $(n \times 1)$ vector of generalised forces exerted by the end-effector on the environment and measured by the wrist force sensor.

For non-redundant robots (1) can be written in cartesian space as:

$\boldsymbol{M}_{x}(x) \ddot{x}+\boldsymbol{c}_{x}(x, \dot{x}) \dot{x}+\boldsymbol{g}_{x}(x)+\boldsymbol{d}_{x}(\dot{x})=\boldsymbol{f}_{\boldsymbol{c}}-\boldsymbol{f}_{\boldsymbol{e}}$

where $x$ is a six-dimensional vector representing the position and orientation of the manipulator end-effector, and the terms of (2) are well known and are given in the literature as in Spong and Vidyasagar (Spong, 1989).

Throughout this paper, a linear spring-damper is adopted for the environment model, according to:

$\boldsymbol{f}_{e}=\boldsymbol{K}_{e}\left(x-x_{e}\right)+\boldsymbol{B}_{e} \dot{x}$ 


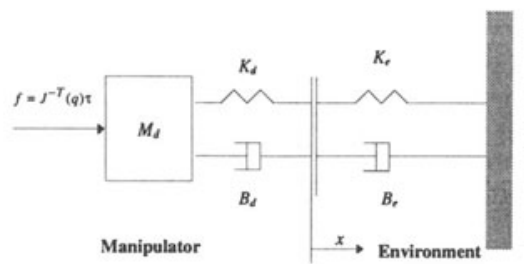

Figure 11 D.O.F. cartesian manipulator: $B_{e}, K_{e}$ - environment parameters. $M_{d}$, $B_{d}, K_{d}$ - Desired robot impedance parameters.

where $\boldsymbol{K}_{e}$ and $\boldsymbol{B}_{e}$ are the environment stiffness and damping matrices, respectively, and $x_{e}$ corresponds to the environment position.

\section{HYBRID IMPEDANCE APPROACH}

The design of the hybrid impedance controller is accomplished in the task space. The task space is splited into two independently controlled subspaces, an impedance controlled subspace and a force controlled subspace. The object of the hybrid impedance controller is to design an algorithm for each subspace such that the dynamics exhibited by the system are replaced by better desirable dynamics. Figure 1 represents the 1-DOF cartesian manipulator case.

In the force controlled subspace, a desired force trajectory must be followed (Bickel, 1995). Additionally, in order to reduce impact forces when the manipulator loses and then regains contact with the environment, low velocities are desirable even in the presence of large force errors. According to Figure 1 the target dynamics are given by:

$\boldsymbol{M}_{d} \ddot{x}+B_{d} \dot{x}-f_{d}=-f_{e}$

The desired reference acceleration for the force controlled subspace which guarantees the desired force following is taken by solving (4) in respect to the acceleration, leading to:

$\ddot{x}_{t f}=M_{d}^{-1}\left(-B_{d} \dot{x}+f_{d}-f_{e}\right)$

Therefore, if this acceleration can be tracked then the desired dynamics given in (4) can be accomplished.

The objective of the controller in the impedance controlled subspace is to track a desired position trajectory in the absence of any forces implied by the environment and, moreover, to react with a desired impedance whenever the presence of such environmental forces exist (Bickel, 1995), as shown in Figure 1. The dynamics in this subspace can be represented by

$M_{d}\left(\ddot{x}_{d}-\ddot{x}\right)+B_{d}\left(\dot{x}_{d}-\dot{x}\right)+K_{d}\left(x_{d}-x\right)=f_{e}$ 


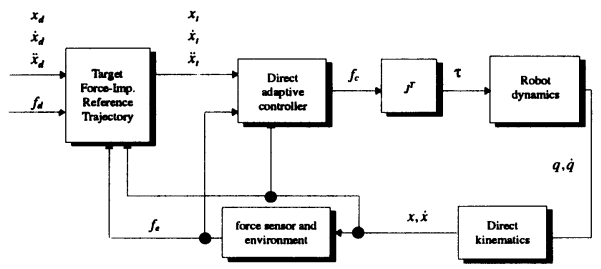

Figure 2 Direct adaptive control block diagram.

which leads to the target reference acceleration for the impedance controlled subspace

$\ddot{x}_{t i}=\ddot{x}_{d}+\boldsymbol{M}_{d}^{-1}\left[\boldsymbol{B}_{d}\left(\dot{x}_{d}-\dot{x}\right)+\boldsymbol{K}_{d}\left(x_{d}-x\right)-\boldsymbol{f}_{e}\right]$

The outer hybrid impedance control loop provides the target acceleration $\ddot{x}_{t}=\left[\begin{array}{ll}\ddot{x}_{t f} & \ddot{x}_{t i}\end{array}\right]^{T}$ to the inner control loop which is designed to linearize the robot dynamics and track $\ddot{x}_{t}$. Given the reference acceleration, we can obtain a target force/impedance reference trajectory, by numerical integration of $\ddot{x}_{t}$, with a proper set of initial conditions, i.e. $x_{d}(0)=x_{t}(0)$ and $\dot{x}_{d}(0)=\dot{x}_{t}(0)$. The target force/impedance trajectory $x_{r e f}=\left[\begin{array}{lll}x_{t} & \dot{x}_{t} & \ddot{x}_{t}\end{array}\right]^{T}$ coincides with the desired tracking trajectory over the unconstrained motion duration, because $\boldsymbol{f}_{e}$ is zero. However, when the robot end-effector reaches the surface it may differ considerably from the desired trajectory due to the environmental forces.

\section{ADAPTIVE CONTROLLERS}

\subsection{Direct adaptive controller}

In this control approach, a non-model based cartesian algorithm generates the control action $\boldsymbol{f}_{c}$ and the required torques to the robot, in order to achieve the desired force/impedance characteristics (Colbaugh, 1995). A block diagram of the hybrid impedance control structure with a direct adaptive controller (DAC), is given in Figure 2.

Consider the following auxiliary vectors:

$\sigma=\dot{e}_{t}+\Lambda e_{t}$

$\dot{x}_{r}=\dot{x}_{t}+\Lambda e_{t}$

where $\dot{x}_{r}$ is a "modified velocity", $\sigma$ is a "weighted position-velocity error" where $e_{t}=$ $x_{t}-x, \dot{e}_{t}=\dot{x}_{t}-\dot{x}$ are the target position and velocity errors, respectively, and $\Lambda$ is a positive scalar constant. Consider the following control law:

$\boldsymbol{f}_{c}=\boldsymbol{A}(t) \ddot{x}_{r}+\boldsymbol{B}(t) \dot{x}_{r}+\boldsymbol{f}_{a}(t)+\boldsymbol{f}_{e}+\left[\boldsymbol{K}_{1}+\boldsymbol{K}_{a}(t)\right] \sigma$

where $\boldsymbol{K}_{1}$ is a positive diagonal gain matrix. The adaptive gains $f_{a}(t) \in \Re^{\eta}, \boldsymbol{A}(t) \in \Re^{n \times \eta}$, 
$\boldsymbol{B}(t) \in \Re^{n \times \eta}$ and $\boldsymbol{K}_{a}(t) \in \Re^{n \times \eta}$, where $\eta$ is the number of coordinates in task space, are updated with the following laws:

$$
\begin{aligned}
& \boldsymbol{f}_{a}(t)=\boldsymbol{f}_{a}(0)-\int_{0}^{t} \alpha_{1} \boldsymbol{f}_{a} d t+\int_{0}^{t} \beta_{1} \sigma d t \\
& \boldsymbol{A}(t)=\boldsymbol{A}(0)-\int_{0}^{t} \alpha_{2} \boldsymbol{A} d t+\int_{0}^{t} \beta_{2} \sigma \ddot{x}_{r}^{T} d t \\
& \boldsymbol{B}(t)=\boldsymbol{B}(0)-\int_{0}^{t} \alpha_{3} \boldsymbol{B} d t+\int_{0}^{t} \beta_{3} \sigma \dot{x}_{r}^{T} d t \\
& \boldsymbol{K}_{a}(t)=\boldsymbol{K}_{a}(0)-\int_{0}^{t} \alpha_{4} \boldsymbol{K}_{a} d t+\int_{0}^{t} \beta_{4} \sigma \sigma^{T} d t
\end{aligned}
$$

In (11-14), the $\beta_{i}$ are positive scalar constants and the $\alpha_{i}$ are functions of the form $\alpha_{i}=\alpha_{i 0}+\alpha_{i 1}\|\dot{x}\|$, where $\alpha_{i 0}, \alpha_{i 1}$ are positive scalar constants (Colbaugh, 1995).

\subsection{Passivity-based adaptive controller}

In this control approach (Siciliano, 1996), the following model-based control law is considered

$\boldsymbol{f}_{c}=\hat{\boldsymbol{M}}_{x}(x) \ddot{x}_{r}+\hat{\boldsymbol{c}_{x}}(x, \dot{x}) \dot{x}_{r}+\hat{\boldsymbol{g}_{x}}(x)+\boldsymbol{f}_{e}+\boldsymbol{K}_{D}\left(\dot{x}_{r}-\dot{x}\right)$

where $\hat{\boldsymbol{M}}_{x}, \hat{\boldsymbol{c}_{x}}, \hat{\boldsymbol{g}_{x}}$ are the estimates of $\boldsymbol{M}_{x}, \boldsymbol{c}_{x}, \boldsymbol{g}_{x}$, respectively, $\dot{x}_{r}, \ddot{x}_{r}$ are $(n \times 1)$ reference vectors and $\boldsymbol{K}_{D}$ is a positive definite matrix of proportional-derivative type. It is assumed that $\hat{\boldsymbol{c}_{x}}$ is skew-symmetric and that $\hat{\boldsymbol{M}}_{x}, \hat{\boldsymbol{c}_{x}}, \hat{\boldsymbol{g}_{x}}$ have the same functional form of $\boldsymbol{M}_{x}, \boldsymbol{c}_{x}, \boldsymbol{g}_{x}$, with a $(m \times 1)$ vector of estimated parameters $\hat{p}$. Then, using the property of linearity of the dynamic model (2) in terms of a proper set of manipulator and load constant parameters, i.e.

$\boldsymbol{M}_{x}(x) \ddot{x}+\boldsymbol{c}_{x}(x, \dot{x}) \dot{x}+\boldsymbol{g}_{x}(x)=\boldsymbol{W}_{x}(x, \dot{x}, \ddot{x}) p$

where $\boldsymbol{W}_{x}(x, \dot{x}, \ddot{x})$ is an $(n \times m)$ regressor matrix, the control law (15) can be given by

$\boldsymbol{f}_{c}=\boldsymbol{W}_{x}\left(x, \dot{x}, \dot{x}_{r}, \ddot{x}_{r}\right) \hat{p}+\boldsymbol{f}_{e}+\boldsymbol{K}_{D}\left(\dot{x}_{r}-\dot{x}\right)$

Combining (2) and (17) leads to

$\boldsymbol{M}_{x}(x) \dot{e}+\boldsymbol{c}_{x}(x, \dot{x}) e+\boldsymbol{K}_{D} e=\boldsymbol{W}_{x}\left(x, \dot{x}, \dot{x}_{r}, \ddot{x}_{r}\right) \tilde{p}$

where $\tilde{p}=\hat{p}-p, \dot{x}_{r}=\dot{x}_{t}+e$ and $\ddot{x}_{r}=\ddot{x}_{t}+\dot{e}$. In this approach, the error $e$ is related to the force and position errors (Siciliano, 1996) by the following equation:

$e=\Delta \dot{x}+\lambda_{1} \Delta x+\lambda_{2} \int_{0}^{t} \Delta \boldsymbol{f} d \delta$ 


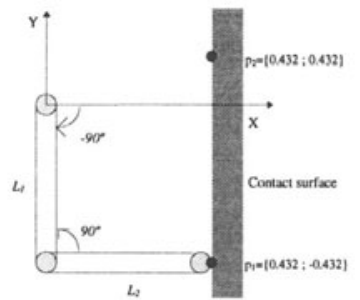

Figure 3 Two link manipulator in vertical plane and environment.

where $\Delta x=x_{t}-x, \Delta \boldsymbol{f}=\boldsymbol{f}_{d}-\boldsymbol{f}_{e}$ and $\lambda_{1}, \lambda_{2}>0$. Then, we obtain

$\dot{x}_{r}=\dot{x}_{t}+\lambda_{1} \Delta x+\lambda_{2} \int_{0}^{t} \Delta f d \delta$

$\ddot{x}_{r}=\ddot{x}_{t}+\lambda_{1} \Delta \dot{x}+\lambda_{2} \Delta f$

Applying the equations that relate the dynamics in the joint space and in the task space for non-redundant manipulators (Spong, 1989), and from (15), finally leads to the control law in joint space

$\tau=\boldsymbol{W}\left(q, \dot{q}, \dot{q}_{r}, \ddot{q}_{r}\right) \hat{p}+\boldsymbol{K}_{D} \boldsymbol{J}^{T}(q) \boldsymbol{J}(q)\left(\dot{q}_{r}-\dot{q}\right)+\boldsymbol{J}^{T} \boldsymbol{f}_{e}$

The parameter estimate update law in the joint space (Siciliano, 1996), is given by

$\dot{\hat{p}}=\Gamma^{-1} \boldsymbol{W}^{T}\left(q, \dot{q}, \dot{q}_{r}, \ddot{q}_{r}\right)\left(\dot{q}_{r}-\dot{q}\right)$

\section{SIMULATION RESULTS}

In this section, the adaptive hybrid impedance control schemes presented in Section 4 are applied to an industrial robot through computer simulation. The robot chosen for this simulation study is the 2-DOF PUMA 560 planar robot which is acting in the vertical plane according to Figure 3. The numerical values for the link parameters of the robot under study are $m_{1}=15.91 \mathrm{Kg}, m_{2}=11.36 \mathrm{Kg}, l_{1}=l_{2}=0.432 \mathrm{~m}$, which resembles the original parameters of links 2 and 3 of the Unimation PUMA 560 arm. Throughout the simulations, the constant time steps of 1 and $2 \mathrm{~ms}$ were used in both controller implementations, while the dynamic model of the robot is simulated in the MATLAB/SIMULINK environment using the Runge-Kutta fourth order integration method.

In this study a frictionless contact surface modelled as in (3) is placed in a vertical position of the robot workspace at $x_{e}=0.432 \mathrm{~m}$, as shown in Figure 3 . It is considered that in all the simulations the end-effector maintains the contact with the surface during the execution of the complete task.

Both control schemes are tested considering an end-effector reference position trajectory from $p_{1}=\left[\begin{array}{ll}0.432 & -0.432\end{array}\right]$ to $p_{2}=\left[\begin{array}{ll}0.432 & 0.432\end{array}\right]$ with a cycloidal reference force profile. The 

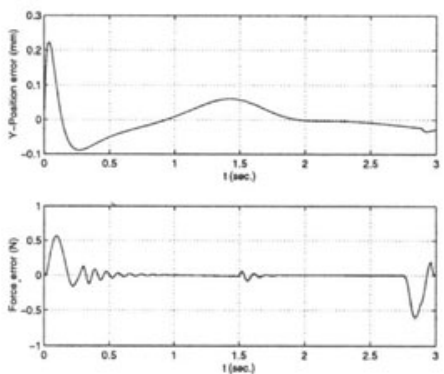

(a) Position and contact force errors


(b) Position and contact force errors

Figure 4 a) Position and contact force errors (DAC). b) Position and contact force errors (IAC). (Note: Sampling time $T_{s}=2 \mathrm{~ms}$ ).

impedance parameters in (6) are defined as $\boldsymbol{M}_{\boldsymbol{d}}=\operatorname{diag}\left[\begin{array}{ll}2.5 & 2.5\end{array}\right], \boldsymbol{K}_{\boldsymbol{d}}=\operatorname{diag}\left[\begin{array}{ll}250 & 250\end{array}\right]$ and the elements of $\boldsymbol{B}_{\boldsymbol{d}}$ are set to provide critical damping in the impedance model. In order to test the robustness of the force control schemes to environmental model uncertainties, a following time varying stiffness profile, was considered:

$\boldsymbol{K}_{e}= \begin{cases}10000+1000 \sin (\pi t / 2) & \text { for } 0<t<2 \\ 10000 e^{(-0.25(t-2))} & \text { for } 2 \leq t<3\end{cases}$

and $\boldsymbol{B}_{e}=\operatorname{diag}\left[\begin{array}{ll}5 & 5\end{array}\right]$. For the direct adaptive controller, the constant parameters are set as $\boldsymbol{K}_{1}=\operatorname{diag}[35003500], \Lambda=30$ and the adaptive gains $\boldsymbol{f}_{a}, \boldsymbol{A}, \boldsymbol{B}$ and $\boldsymbol{K}_{\boldsymbol{a}}$ as defined in Section 4 are set initially to zero, with $\alpha_{i 0}=0.001, \beta_{i}=1, i=1, \ldots, 4$. For the passivitybased adaptive controller (IAC), the values of the matrices $K_{D}, \Gamma$ and the initial values of the estimated parameter vector $\hat{p}$, are:

$\boldsymbol{K}_{D}=\operatorname{diag}[1500 \quad 1500]$

$\Gamma=\operatorname{diag}\left[\begin{array}{lllll}0.02 & 0.02 & 0.02 & 0.002 & 0.002\end{array}\right]$

$\hat{p}(0)=\left[\begin{array}{lllll}3.0 & 1.6 & 0.4 & 70.0 & 12.0\end{array}\right]$

with $\lambda_{1}=30, \lambda_{2}=0.15$. The results presented in Figure 4, show that both adaptive controllers have good position and force tracking capability for the given trajectory. The results in Table 1 show also that the hybrid impedance approach with passivity-based adaptive algorithm (IAC) has a better performance, with position and force errors less than $0.3 \mathrm{~mm}$ and $0.6 \mathrm{~N}$, respectively, at a cost of a higher number of MATLAB floatingpoint operations (FLOPS). Moreover, the IAC algorithm reveal to be more robust to a moderate increase in the sampling time (Ex: $T_{s}=4 \mathrm{~ms}$ ). 
Table 1 Sum squared errors of $y$ coordinate and contact force $\boldsymbol{f}_{e}$.

\begin{tabular}{lrrrr}
\hline & DAC & IAC & DAC & IAC \\
\hline & $T_{s}=1 \mathrm{~ms}$ & $T_{s}=1 \mathrm{~ms}$ & $T_{s}=2 \mathrm{~ms}$ & $T_{s}=2 \mathrm{~ms}$ \\
\hline $\operatorname{SSE}(\boldsymbol{y})$ & $5.45 \times 10^{-6}$ & $6.68 \times 10^{-6}$ & $3.48 \times 10^{-6}$ & $4.04 \times 10^{-6}$ \\
$\mathrm{SSE}(\dot{\boldsymbol{y}})$ & 0.0028 & 0.0034 & 0.0024 & 0.0018 \\
$\mathrm{SSE}\left(\boldsymbol{f}_{e}\right)$ & 56.11 & 45.61 & 27.65 & 22.71 \\
$\#$ FLOPS & $1.55 \times 10^{6}$ & $2.93 \times 10^{6}$ & $0.77 \times 10^{6}$ & $1.46 \times 10^{6}$ \\
\hline
\end{tabular}

\section{CONCLUSIONS}

In this article an adaptive hybrid impedance control strategy for robot manipulators is presented. In order to test the performance of the proposed control scheme two distinct adaptive control algorithms were tested in the inner control loop. The study has shown good results and demonstrated some of the potential applications of the adaptive hybrid impedance control in robotic tasks that require explicit force tracking capability in an environment with a time varying stiffness.

Future research will cover the improvement of the proposed scheme, stability analysis, and an accurate study of the impact situation in the context of the hybrid impedance control approach.

\section{REFERENCES}

Neville Hogan. Impedance control: an approach to manipulation: Part I-II-III. Journal of Dynamic Systems, Measurement, and Control, 107:1-24, March 1985.

M. Raibert and J. Craig. Hybrid position/force control of manipulators. Journal of Dynamic Systems, Measurement, and Control, 102:126-133, June 1982.

R. J. Anderson and M. W. Spong. Hybrid impedance control of robotic manipulators. IEEE Transactions on Robotics and Automation, 4:549-556, October 1988.

J. Craig and S. S. Sastry. Adaptive control of mechanical manipulators. Proc. IEEE International Conference on Robotics and Automation, pages 190-195, 1986.

W. S. Lu and Q. H. Meng. Impedance control with adaptation for robotic manipulators. IEEE Transactions on Robotics and Automation, 7:408-412, June 1991.

R. Colbaugh, H. Seraji, and K. Glass. Adaptive compliant motion control for dextrous manipulators. Journal of Robotic Systems, 14:270-280, June 1995.

Bruno Siciliano and Luigi Villani. A passivity-based approach to force regulation and motion control of robot manipulators. Automatica, 36, 1996.

Mark W. Spong and M. Vidyasagar. Robot dynamics and control. John Wiley and Sons, Inc., Republic of Singapure, 1989.

R. J. Bickel and M. Tomizuka. Disturbance observer based hybrid impedance control of robotic manipulators. American Control Conference, June 1995. 